\title{
THE SYSTEMATIC SEROLOGY OF NEISSERIA GONORRHOEAE: ANTIGENS ASSOCIATED WITH PATHOGENESIS IN NEISSERIA SPP. FROM MAN
}

\author{
K. U. SaIKh and F. KathleEn Bhattacharyya
}

Department of Bacterial Immunology, Indian Institute of Chemical Biology, 4, Raja S.C. Mullick Road, Calcutta-700032, India

\begin{abstract}
SUmmary. Sonicates of eight Neisseria species from man were analysed in a micro-Ouchterlony double-diffusion absorption assay in comparison with a gonococcal reference antiserum-antigen system. Five major gonococcal precipitin zones were identified which comprised genus-, species- and type-specific components. One antigen was found in all strains of three species with pathogenic capability $-N$. gonorrhoeae, $N$. meningitidis and $N$. flavescens. It was not detected in $N$. lactamica, $N$. pharyngis, $N$. elongata, $N$. cinerea or $N$. catarrhalis.
\end{abstract}

\section{INTRODUCTION}

In gonococcal research, the quest for specific antigens for use in serodiagnosis, prophylaxis and serotyping has a long history. The trial and error approach to these problems has failed, yet there have been relatively few efforts to obtain systematic serological information about the genus Neisseria. Early work was compromised by the instability of the gonococcus in culture. Intracellular genus-specific antigens (Danielsson, 1965a), both surface (Johnston, Holmes and Gotschlich, 1976) and internal (Geizer, 1975) type-specific antigens and also surface-located subgeneric antigens (Johnston et al., 1976) have been described. Since about 1970 there have been great improvements in methods for the culture of stable gonococci of known infectivity (Kellogg et al., 1968) which may be considered antigenically "complete". Taking advantage of these techniques we undertook a systematic investigation of the Neisseria spp. from man, with a view to generating basic serological information about the gonococcus.

\section{MATERIALS AND METHODS}

Bacterial strains. The organisms used in this study were: (a) 11 representative gonococcal strains from local and international sources; local strains were selected on the basis of their susceptibility to nine antibiotics (Gupta et al., 1982) because this is known to correlate with other epidemiological markers (Bygdeman, 1981); (b) NCTC reference strains of four serogroups of N. meningitidis; (c) six NCTC strains of human commensal Neisseria (National Collection of Type Cultures, 175 Colindale Avenue, London NW9 5HT).

Identity of strains was confirmed by standard bacteriological methods including sugar 
utilisation tests (Young, Peterson and McDonald, 1976), polysaccharide production from sucrose (Catlin, 1970) and immunofluorescence assay with FA $N$. gonorrhoeae antiserum (Difco). The cultures were grown routinely in GC Medium agar base (Difco) with defined supplement (Kellogg et al., 1963) for $20 \mathrm{~h}$ in a humid candle jar at $36^{\circ} \mathrm{C}$. Colony morphology was typed and cultures were lyophilised by methods described previously (Saikh and Bhattacharyya, 1983).

Antigens and antisera. Cells were harvested in cold $50 \mathrm{~mm}$ Tris- $\mathrm{HCl}$ buffer $p \mathrm{H} 7.5$ to a turbidity equivalent of $2000 \mathrm{Klett}$ units ( $540 \mathrm{~nm}$ filter). Antigens for immunodiffusion were cells which had been lysed in the MSE 100 Ultrasonic Disintegrator for a total of $10 \mathrm{~min}$ in ice at amplitude 7 and a frequency of $20 \mathrm{Kcycles} / \mathrm{s}$. Sonicates were preserved in divided portions at $-18^{\circ} \mathrm{C}$. Cell suspensions fixed with formalin $0.5 \% \mathrm{v} / \mathrm{v}$ and diluted 1 in 10 in phosphate-buffered

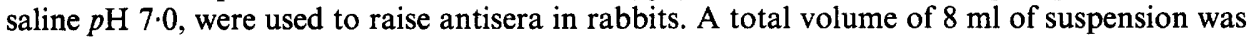
administered in eight graded, bi-weekly injections, initially by the intramuscular route and subsequently by the intravenous route. Preimmune serum was obtained from each animal.

Immunodiffusion (ID). Gels of $2 \mathrm{~mm}$ thickness were set and cut on microscope slides using the Gelman ID kit with $3 \mathrm{~mm}$ cutters. The gel was Oxoid Agar No. 1, 1\%w/v; barbital buffer $p \mathrm{H}$ $7 \cdot 4$, ionic strength $0 \cdot 15$; polyethylene glycol $6000,1 \cdot 5 \% \mathrm{w} / \mathrm{v}$; sodium azide $0.2 \% \mathrm{w} / \mathrm{v}$. The wells were filled with reactants and the slides incubated at $36^{\circ} \mathrm{C}$ for $48 \mathrm{~h}$ in humidity chambers before recording the reactions. After development, the slides were washed, dried and stained with amido black (Crowle, 1973).

The antigen and antiserum preparations were tested by direct comparison and also by absorption analysis. Absorption of antisera with intracellular as well as cell-surface components was done by prediffusion of absorbing sonicate in the antiserum well for $2 \mathrm{~h}$ before adding the reactants. A double application of the absorbing antigen was done if absorption was found incomplete. Absorption of antibody to only the cell-surface antigens was done with formalinfixed cells: $1 \mathrm{~g}$ wet weight of cells was mixed with $1 \mathrm{ml}$ of antiserum for $5 \mathrm{~h}$ at $4^{\circ} \mathrm{C}$ (Danielsson, $1965 b$ ). The serum was then clarified by centrifugation.

\section{RESULTS}

\section{Rabbit response to immunisation}

Individual sera from ten rabbits were tested before immunisation and were found to be unreactive in ID with gonococcal sonicates. Considerable variation was observed
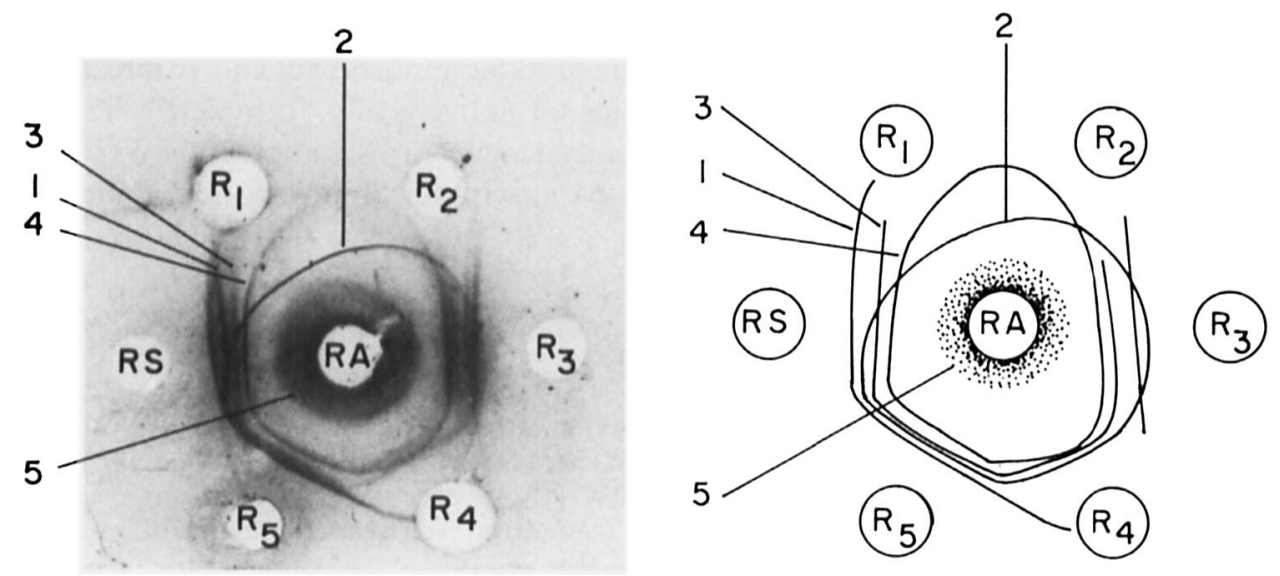

FIG. 1.-Variation in rabbit response to immunisation with gonococcal whole-cell antigen demonstrated by immunodiffusion analysis: $R_{1}, R_{2}, R_{3}, R_{4}, R_{5}=$ antisera to gonococcal strain $N 133$ from five different rabbits; $\mathrm{RS}=\mathrm{N} 133$ pooled reference antiserum; $\mathrm{RA}=\mathrm{N} 133$ reference antigen sonicate; $1,2,3,4$ and $5=$ designation of precipitin bands in reference pattern. 
in the rabbit antibody response to formalin-fixed gonococcal cells with the four gonococcal strains investigated. Five rabbits were used for antiserum preparation against the gonococcal reference strain N133. Their antisera showed two kinds of response when examined by double-diffusion analysis with the $\mathrm{N} 133$ reference sonicate antigen (RA, fig. 1). Precipitin lines which were prominent with some sera were absent in others; e.g., antisera from rabbits R1, R2 and R4 gave precipitin reactions with only two of the five antigen complexes detected by sera from rabbits R3 and R5. This response was mainly to the type-specific complex 2 . Because of this variability a N133 reference antiserum (RS) was prepared by pooling sera to gain maximum resolution of precipitin bands. These bands were designated 1 to 5 on the basis of their proximity to the antiserum well. Band position, particularly of component 2 was subject to some variation because of slide variables such as thinness of the agar at the slide edge (compare fig. 3 and fig. 5).

\section{Analysis of gonococcal sonicates}

Gonococcal sonicates gave reproducible precipitin patterns in ID irrespective of colony type and preparation batch. Although pure virulent-colony-type preparations (T1 or T2) cannot be obtained because of the high degree (c. $20 \%$ ) of segregation on subculture, T1- and T2-enriched sonicates were compared with sonicates of pure avirulent cultures (T3 and T4). No qualitative differences in band patterns were detected. Where possible, mixed sonicates of all four colony types were used; they were obtained by plating each type separately and harvesting them in equal proportions. Avirulent type 5- segregants (Jephcott and Reyn, 1971) and opacity variants (Swanson, 1978) were not examined.

All the precipitin bands except for band 3, were found to consist of multiple components in some reaction conditions, e.g., in reactions with other sera and in absorption analysis (see below). The precipitin bands are, therefore, referred to as zones. When sonicates from several gonococcal isolates were compared with the N133 reference system, some quantitative and qualitative differences were evident (fig. 2),
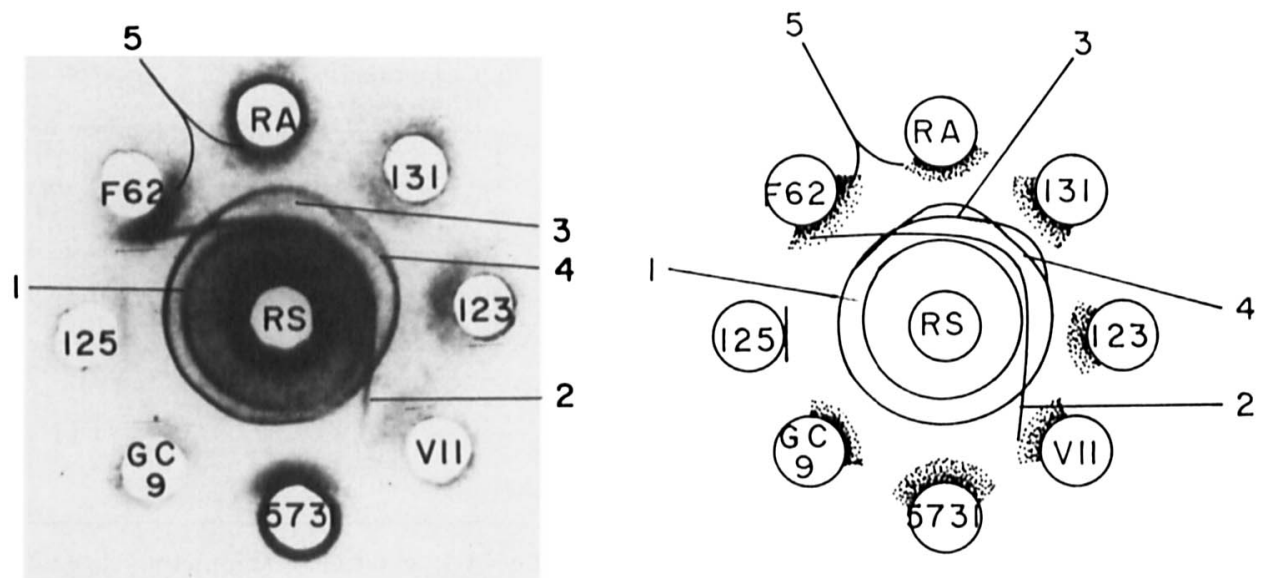

FIG. 2.-Reactivity of gonococcal sonicates with reference anti-serum N133: Outer wells = antigen sonicates of Calcutta isolates N123, N125 and N131 and international strains F62, GC9, WHO VII and 5731. 

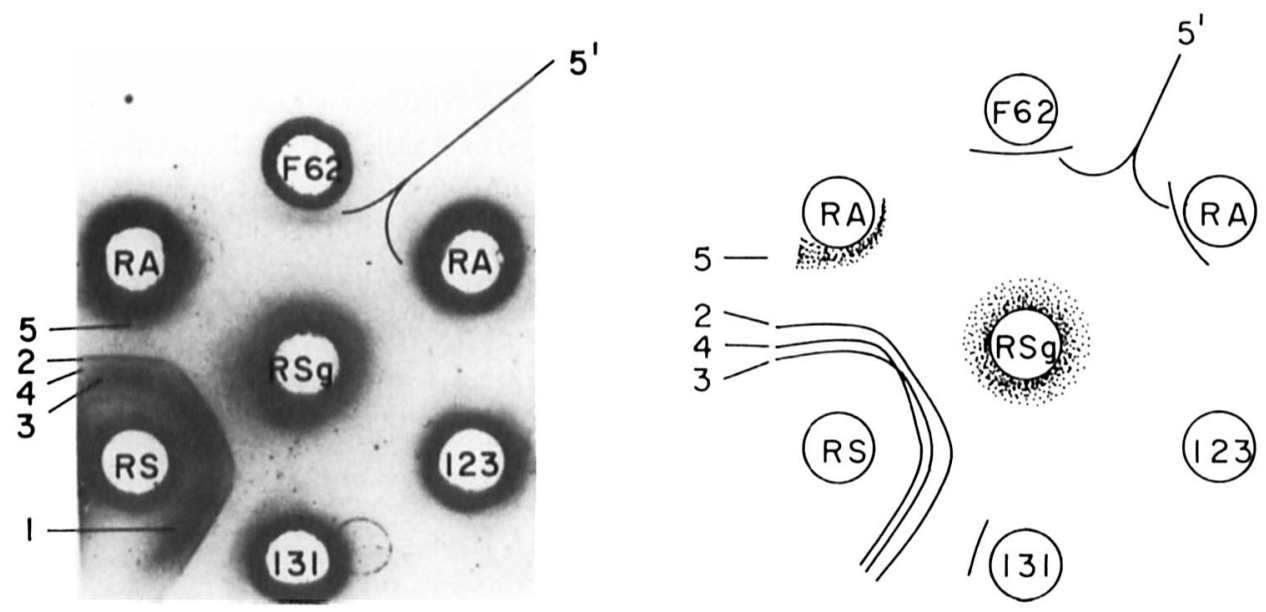

FIG. 3- - Type-specific gonococcal antigens shown by pre-diffusion absorption with gonococcal sonicate: $\mathrm{N} 131, \mathrm{~N} 123, \mathrm{~F} 62=$ gonococcal antigen sonicates; RSg = reference antiserum absorbed by prediffusion of N131 sonicate; $5^{\prime}=$ strain specific component of band 5 .

e.g., N133 (RA) was not a good producer of zone 5. The distribution of the five zones among gonococci is compiled in table I. Zones 1, 3, and 4 showed complete identity in all gonococci (fig. 2). The prominent zone 2 was strain-specific, and occurred irrespective of antibiotic-resistance type. The majority of the Calcutta strains contained this component whereas only one of five international strains did so (fig. 2; table I). These results were confirmed by intragel absorption analysis where the

\section{TABLE I}

Cross-reactivity of the $N 133$ gonococcal reference antiserum with sonicates of 11 gonococcal strains

\begin{tabular}{|c|c|c|c|c|c|c|c|}
\hline \multicolumn{3}{|r|}{ Test antigen } & \multicolumn{5}{|c|}{$\begin{array}{l}\text { Precipitin bands formed with N133 reference } \\
\text { antiserum }\end{array}$} \\
\hline \multirow[b]{2}{*}{ Strain } & \multirow[b]{2}{*}{ Source } & \multirow{2}{*}{$\begin{array}{l}\text { Antibiotic resistance type* } \\
\text { and other characteristics }{ }^{\dagger}\end{array}$} & \multicolumn{4}{|c|}{$\begin{array}{l}\text { Inner zone (anti- } \\
\text { serum well) }\end{array}$} & \multirow{2}{*}{ Outer zone (antigen well) } \\
\hline & & & 1 & 2 & 3 & 4 & \\
\hline N123 & Calcutta & Ery, Pc, Cl, Tc, Sm, Su, Cm; T1-T4 & + & + & + & + & + \\
\hline N125 & Calcutta & Amp, Pc, Cl, Sm; T4 & + & - & + & + & + \\
\hline N131 & Calcutta & All sensitive; $\mathrm{T} 1-\mathrm{T} 4$ & + & + & + & + & + \\
\hline N133 & Calcutta & Ery, Pc, Cl, Tc, Sm; Tl-T4 & + & + & + & + & + \\
\hline N179 & Calcutta & Amp, $\mathrm{Pc}, \mathrm{Cl}, \mathrm{Tc}, \mathrm{Sm}, \mathrm{Su} ; \mathrm{T} 4$ & + & + & + & + & + \\
\hline $\mathrm{N} 213$ & Calcutta & Ery, Pc, Cl, Amp, Tc, Sm, Su; T4 & + & - & + & + & + \\
\hline F62 & USA & All sensitive; T1-T4; Pro & + & - & + & + & + \\
\hline GC9 & USA & Not done; T1-T4; Dark & + & - & + & + & + \\
\hline Н 8793 & Britain & Ery, Pc, Cl, Amp, Tc, Sm, Su; T4; $\beta$ & + & + & + & + & + \\
\hline 5731 & Sweden & Pc, Amp, Cl, Sm; T4; $\beta$ & + & - & + & + & + \\
\hline WHO VII & Sweden & All sensitive; T4 & + & - & + & + & + \\
\hline
\end{tabular}

* Resistance to erythromycin $=$ Ery; penicillin $=\mathrm{Pc}$; cloxacillin $=\mathrm{Cl}$; ampicillin $=\mathrm{Amp}$; tetracycline $=\mathrm{Tc}$; streptomycin $=\mathrm{Sm}$; sulphamethoxazole $=\mathrm{Su}$; chloramphenicol $=\mathrm{Cm}$.

$\uparrow$ T1-T4 denotes the mixture of the four different morphological colony types in the sonicates. Pro $=$ proline auxotroph; Dark $=$ dark colony variant; $\beta=$ presence of $\beta$-lactamase. 
TABLE II

Cross-reactivity of $N 133$ gonococcal reference antiserum with sonicates of other Neisseria spp.

\begin{tabular}{|c|c|c|c|c|c|c|}
\hline \multirow{2}{*}{\multicolumn{2}{|c|}{ Test antigen }} & \multicolumn{5}{|c|}{$\begin{array}{l}\text { Precipitin bands formed with N133 reference } \\
\text { antiserum }\end{array}$} \\
\hline & & \multicolumn{4}{|c|}{ Inner zone (antiserum well) } & \multirow{2}{*}{$\begin{array}{c}\text { Outer zone (antigen well) } \\
5\end{array}$} \\
\hline Species & Strain no. & 1 & 2 & 3 & 4 & \\
\hline N. gonorrhoeae & N133 & + & + & + & + & + \\
\hline$N$. meningitidis (Group A) & NCTC10025 & + & - & + & + & + \\
\hline N. meningitidis (Group B) & NCTC 10026 & + & - & + & + & + \\
\hline N. meningitidis (Group C) & NCTC 8554 & + & - & + & + & + \\
\hline N. meningitidis (Group D) & NCTC9714 & + & - & + & + & + \\
\hline N. lactamica & NCTC10617 & + & - & + & - & - \\
\hline N. flavescens & NCTC 8263 & + & - & + & + & - \\
\hline N. pharyngis & NCTC 4590 & + & - & + & - & - \\
\hline N. elongata & NCTC 10660 & + & - & + & - & - \\
\hline N. cinerea & NCTC 10294 & + & - & + & - & - \\
\hline N. catarrhalis & NCTC 4103 & - & - & - & - & - \\
\hline
\end{tabular}

gonococcal sonicate was allowed to diffuse from the antiserum well before the other reactants were added. Strain-specific components were also revealed in zone 5 (fig. 3 ).

Prediffusion of gonococcal sonicate N131 prevented the subsequent formation of precipitin bands with sera N131 and N123 but not with N133(RA) and F62 with which residual reactions in zone 5 were obtained.

Absorption of the reference serum with intact cells of strain N133 removed precipitins of only zone 5 , indicating that components of zones $1-4$ were located internally in the cell of the reference strain.

\section{Cross-reactivity of other Neisseria spp. with the N133 gonococcal reference system}

Of the 10 strains of $N$. meningitidis and commensal Neisseria spp. tested, various degrees of cross-reactivity with the N133 gonococcal reference system were seen. $N$. catarrhalis did not react. Zones 1 and 3 were present in all the other Neisseria spp, but zone 2 was missing (table II). The presence of zones 1 and 3 was confirmed by intragel absorption. For example, when the gonococcal reference serum (RS) was absorbed by prediffusion with a sonicate of $N$. lactamica, only zones 2,4 and 5 of the reference system remained (fig. 4). No precipitin bands were seen with the $N$. pharyngis sonicate whereas zone 4 was formed with the $N$. flavescens sonicate. Zone 4 was also shared by all four serogroups of meningococci in addition to $N$. flavescens and it was absent from the other Neisseria species (table II). This finding was confirmed by the absorption test (figs 4 and 5). Cross-reactivity in zone 5 was restricted to meningococci and was only partial. These results were confirmed by absorption (fig. 5). In this experiment, prediffusion of $N$. meningitidis serogroup A sonicate prevented the formation of precipitin bands with all the meningococci, whereas residual activity in the gonococcus N133 (RA) was seen in zones 2 and 5. In zone 5, in addition to strain-specific activity, a minor late-developing component remained in all the gonococci tested.

The heterogeneous nature of zones 2 (fig. 5), 4 (fig. 4) and 5 (fig. 3 and 5) was evident in these slides. 

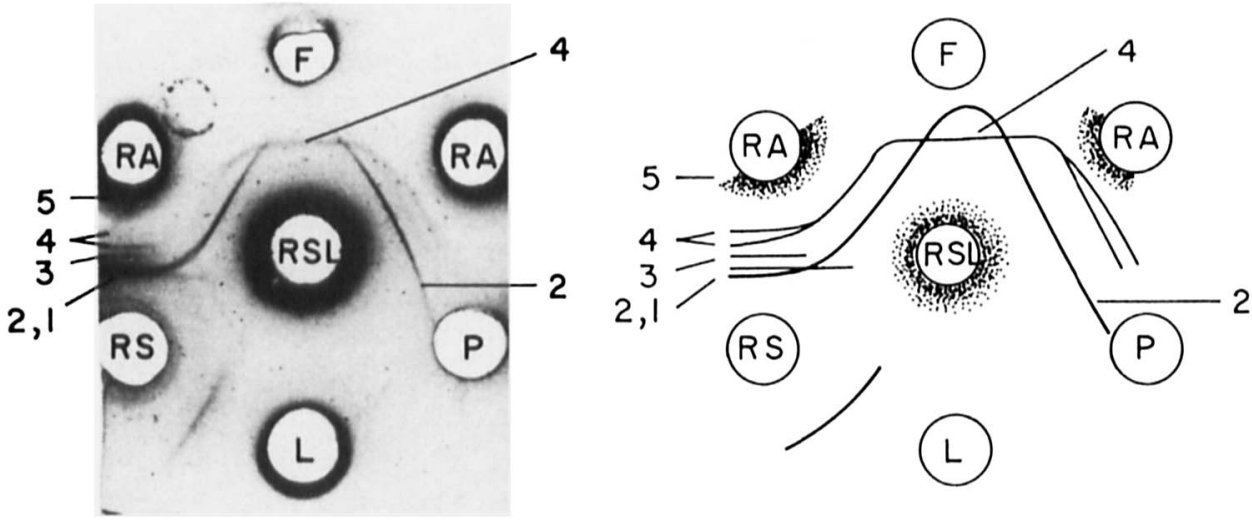

FIG. 4.-Subgeneric group antigens of Neisseria spp. shown by prediffusion absorption of gonococcal reference antiserum with $N$. lactamica:F,P, L and RA=antigens sonicates of $N$. flavescens, $N$. pharyngis, $N$. lactamica and gonococcal reference strain; RSL $=$ reference antiserum absorbed by prediffusion with sonicate of $N$. lactamica.

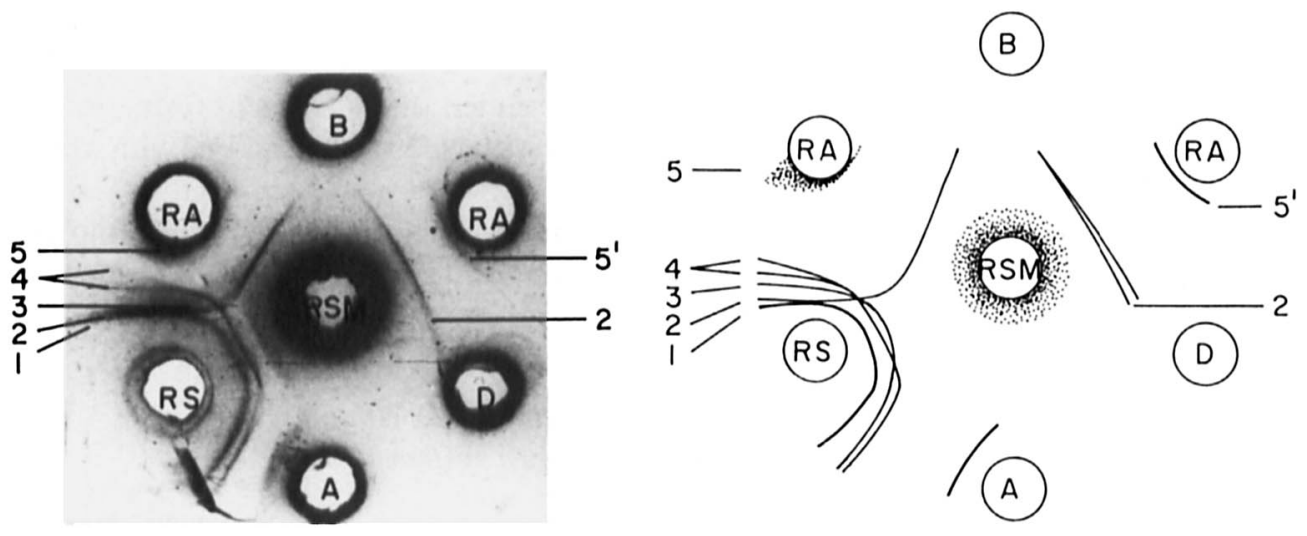

FIG. 5.- Specific gonococcal antigens shown by predifussion absorption analysis of gonococcal reference antiserum with $N$. meningitidis serogroup $\mathrm{A}: \mathrm{B}, \mathrm{D}, \mathrm{A}=$ antigen sonicates of $N$. meningitidis serogroup $\mathrm{B}$, $\mathrm{D}$ and $\mathrm{A}$ respectively; $\mathrm{RSM}=$ reference antiserum absorbed by prediffusion of $N$. meningitidis serogroup A sonicate; $5^{\prime}=$ gonococcus-specific component of band 5 .

\section{DISCUSSION}

With the N133 gonococcal reference serum, it was possible to detect several fundamental inter-relationships between the gonococcus and other Neisseria spp. All the species could be grouped into several hierarchical categories which is in agreement with current views on their classification. $N$. catarrhalis, now classified in the genus Branhamella, was found not to be related to the gonococcus in this assay. Antigens 1 and 3 appeared to be intracellular and present in all the "true" Neisseria spp. tested; they were, therefore, group antigens at the generic level.

Major components of zone 5, which are located at the cell surface, were subgeneric, being common to the pathogens $N$. gonorrhoeae and $N$. meningitidis. Also of interest was subgeneric zone 4 , the components of which were intracellular. Zone 4 was not 
only a feature of $N$. gonorrhoeae and $N$. meningitidis but also $N$. flavescens. This NCTC strain had been the cause of an epidemic of meningitis (Branham, 1930). However, $N$. flavescens is rarely isolated in health or disease; the use of selective media, on which it does not grow, may be a reason for this. Furthermore, it is not easily distinguished from other asaccharolytic species, $N$. cinerea and B. catarrhalis (Knapp et al., 1984), making interpretation of subsequent reports on the isolation of these species unreliable. The strain of $N$. flavescens used in this study, however, was known to have pathogenic capability. It was also investigated in immunoelectrophoresis by Høff and Høiby (1978) and was shown to have a meningococcal component, arc 55, in common with gonococci. Cannon and Black (1982) have also reported a neisserial component, which they associated with pathogenicity, by the use of monoclonal antibody. Pathogenesis in the neisseriae is associated with factors, e.g., the exoenzyme IgA protease, not found in the commensals (Mulks and Plaut, 1978). Zones 4 and 5 may represent similar components which play a role in pathogenesis.

In addition to generic and subgeneric antigens, gonococcal type-specific components were detected in zones 2 and 5. The geographical distribution of the internal cell components in zone 2 amongst gonococci from local and international sources was clear. This class of antigen may prove a useful addition to current serotyping systems (Johnston et al., 1976; Danielsson and Sandstrom, 1979). In future studies, it will be important to identify responder rabbits for raising antisera because, in our series, only two of five animals produced high levels of antibody to the 2 complex, to the exclusion of several other precipitins. The distribution of type-specific elements of zone 5 was less frequent and could not be assessed for epidemiological significance in this limited study. This complex appeared to be similar to the type antigens of Johnston et al. (1976) in cell-surface location, diffusion characteristics and in possessing group and type specificities. A gonococcal species-specific component was also apparent in this zone on prolonged incubation. Further work with cell-wall preparations is necessary to resolve this potentially important antigen complex.

There have been few investigations of whole-cell antigens of the neisseriae, and only those of Danielsson (1965a and $b$ ) were comparable in scope to ours. Our study differed in the use of colony type-defined cell suspensions and in the method of absorption. Danielsson was unable to detect antigens which could be associated with pathogenesis or serotype. Two important reasons for this may have been failure of his absorption technique to remove precipitins to intracellular components, leading to obscurity of reaction patterns, and the over-riding factor of rabbit response to immunisation which was not recognised. The rabbit antibody response was highly variable and was the single most important determinant of antigen resolution in our analysis. K.U.S.

This investigation was partly supported by a R. D. Birla Smarak Kosh Fellowship grant to

\section{REFERENCES}

Branham S E 1930 A new meningococcus-like organism (Neisseria flavescens, $\mathrm{n}$. Sp.) from epidemic meningitis. Public Health Reports 45:845-849.

Bygdeman S 1981 Gonorrhoea in men with homosexual contacts. Serogroups of isolated gonococcal strains related to antibiotic susceptibility, site of infection and symptoms. British Journal of Venereal Disease 57:320-324. 
Cannon J G, Black W J 1982 A monoclonal antibody that binds to the pathogenic Neisseria but not to most non-pathogenic Neisseria species. American Society of Microbiology, Abstracts of the Annual Meeting, $\mathrm{p} 48$.

Catlin B W 1970 Neisseria meningitidis (Meningococcus). In: Blair J E, Lennette E H, Truant J P (eds) Manual of clinical microbiology, American Society of Microbiology, Washington, D.C., p 78.

Crowle A J 1973 Immunodiffusion, 2nd edn, Academic Press, New York, p 517.

Danielsson D 1965a The demonstration of Neisseria gonorrhoeae with the aid of fluorescent antibodies. 3. Studies by immuno-fluorescence and double diffusion-in-gel technique on the antigenic relationship between strains of Neisseria gonorrhoeae. Acta Pathologica et Microbiologica Scandinavica 64:243-266.

Danielsson D $1965 b$ The demonstration of Neisseria gonorrhoeae with the aid of fluorescent antibodies. 4. Studies by immunofluorescence and double diffusion-in-gel technique on the antigenic relationship between Neisseria gonorrhoeae and other Neisseria strains. Acta Pathologica et Microbiologica Scandinavica 64:267-276.

Danielsson D, Sandstrom E 1979 Serology of Neisseria gonorrhoeae. Demonstration of strain specific antigens by immuno-electrophoresis, immunofluorescence and co-agglutination techniques. Acta Pathologica et Microbiologica Scandinavica Section B 87:55-64.

Geizer I 1975 Studies on serotyping of Neisseria gonorrhoeae. Zentralblatt fur Bakteriologie Parasitenkunde Infektionskrankheiten und Hygiene, Abt l Orig A, 232:213-220.

Gupta P, Saikh K U, Ghosh S K, De J, Nandi M, Saha B, Bhattacharyya F K 1982 Modified diffusion assay for detecting antibiotic resistance in Neisseria gonorrhoeae. Indian Journal of Medical Research 75:337-343.

Høff G E, Høiby N 1978 Cross-reactions between Neisseria meningitidis and twenty seven other bacterial species. Acta Pathologica et Microbiologica Scandinavica Section B 86:87-92.

Johnston K H, Holmes K K, Gotschlich E C 1976 The serological classification of Neisseria gonorrhoeae. 1. Isolation of the outer membrane complex responsible for serotypic specificity. Journal of Experimental Medicine 143:741-758.

Jephcott A E, Reyn A 1971 Neisseria gonorrhoeae. Colony variation I. Acta Pathologica et Microbiologica Scandinavica Section B 79:609-614.

Kellogg D S, Peacock W L, Deacon W E, Brown L, Pirkle C I 1963 Neisseria gonorrhoeae. I. Virulence genetically linked to clonal variation. Journal of Bacteriology 85:1274-1279.

Kellogg D S, Cohen C R, Norins L C, Schroeter A L, Reising G 1968 Neisseria gonorrhoeae. II. Colonial variation and pathogenicity during 35 months in vitro. Journal of Bacteriology 96:596-605.

Knapp J S, Totten P A, Mulks M H, Minshew B H 1984 Characterization of Neisseria cinerea, a non-pathogenic species isolated on Martin-Lewis medium selective for pathogenic Neisseria spp. Journal of Clinical Microbiology 19:63-67.

Mulks M H, Plaut A G 1978 IgA protease production as a characteristic distinguishing pathogenic from harmless Neisseriaceae. New England Journal of Medicine 299:973-976.

Saikh K U, Bhattacharyya F K 1983 Variability of media batch and stability of virulent colony morphology Neisseria gonorrhoeae. Indian Journal of Medical Research 77:608-612.

Swanson J 1978 Studies on gonococcus infection. XII. Colony colour and opacity variants of gonococci. Infection and Immunity 19:320-331.

Young H, Paterson I C, McDonald D R 1976 Rapid carbohydrate utilisation test for identification of Neisseria gonorrhoeae. British Journal of Venereal Disease 52:172-175. 